\title{
Second Generation Tyrosine Kinase Inhibitors Combined With Allogeneic Hematopoietic Stem Cell Transplantation Improve the Prognosis of Patients with Chronic Myelogenous Leukemia in Blast Crisis
}

\author{
Zhengping $\mathrm{Yu}^{1}$, Jia-hua Ding ${ }^{{ }^{*}}$, Aining Sun ${ }^{2}$, Zheng $\mathrm{Ge}^{1}$, Baoan Chen ${ }^{1}$ and Wenduo $\mathrm{He}^{1}$ \\ ${ }^{1}$ Department of Hematology, Key Department of Jiangsu Medicine, Zhongda Hospital, Medical School, Southeast University, Nanjing, China \\ ${ }^{2}$ Hematology Division, Suzhou Medical University, Suzhou, China
}

\begin{abstract}
The prognosis for patients with Chronic Myelogenous Leukemia (CML) in Blastic Crisis (BC) is poor, with a median survival of only 3-6 months. Blast crisis (BC) is highly refractory to therapy and has a poor prognosis. To determine the efficacy of TKIs-II combined with allogeneic hematopoietic stem cell transplantation (allo-HSCT) in CML BC, we present four consecutive, recent cases of CML BC in which TKIs-II were used before or after allo-HSCT. Patient 1, a 28-year-old male received a HSCT from a half-matched, related-donor. Patient 2, 3 received an HLAidentical unrelated-donor HSCT. To date, patients 1,2,3 and 4 have survived postprocedure for 22, 23, 21 and 25 months, respectively. We conclude that compared with imatinib, TKIs-II may reduce tumor burden more rapidly and thoroughly when administered before or after allo-HSCT and enhance the graft versus leukemia effect, prolonging the long-term survival of patients. We speculate that GVL and tumor burden are negatively correlated.
\end{abstract}

Keywords: Second generation tyrosine kinase inhibitors; Allogeneic hematopoietic stem cell transplantation; Chronic myelogenous leukemia; Blast crisis

\section{Introduction}

Chronic Myelogenous Leukemia (CML) typically progresses through 3 phases: the Chronic Phase (CP), the Accelerated Phase (AP), and the Blast Crisis (BC). The therapy of blast crisis in patients with CML is a major challenge, with limited chances of success. Historically, therapy for $\mathrm{BC}$ has used combination chemotherapy regimens. This treatment is frequently associated with significant toxicity and mortality, and with short remission durations, and low long-term survival rate. Allogeneic hematopoietic stem cell transplantation (allo-HSCT) induces durable remission in less than $10 \%$ of CML patients with $\mathrm{BC}$ [1]. Even the achievement of complete hematologic remission (CHR) with imatinib before transplantation does not improve the prognosis of CML in $\mathrm{BC}$, probably due to the frequent development of imatinib resistance in BC [2]. Second-generation tyrosine kinase inhibitors (TKIs-II) alone induce CCyR in $20-40 \%$ of patients [3,4]. However, the majority of these patients relapse within 1 year, and the median survival is only 8 months [4]. Thus, although TKIs-II is an effective agent in this setting, it is necessary to improve these results. Few reports [5] are available about the efficacy of TKIs-II combined with allo-HSCT in CML BC. In the current study, we analyzed the effect of treatment with TKIs-II before or after allo-HSCT in four CML patients in BC and discussed the possible mechanisms.

\section{Materials and Methods}

\section{Patients}

Patient 1: A 28-year-old manpresented with priapism in 2009. A complete blood count showed that his White Blood Cell (WBC) count was $444 \times 10^{9} / \mathrm{L}$. A bone marrow aspirate examination revealed $0.01 \%$ myeloid blasts. Fluorescence in situ hybridization (FISH) showed that $90 \%$ of the cells tested were positive for the BCR/ABL fusion gene, and the BCR-ABL: ABL ratio detected by reverse transcription polymerase chain reaction (RT-PCR) was $42 \%$. A diagnosis of Chronic-Phase (CP) CML was made. The patient commenced treatment with $400 \mathrm{mg} /$ day imatinib (Novartis US). After 3 months, the Philadelphia (Ph) chromosome was detected on cytogenetic studies of bone marrow specimens, and the BCR-ABL: ABL ratio detected by RT-PCR was $0.53 \%$. In December 2010, the BCR/ABL fusion gene was detectable on FISH 0/500, and the patient was deemed to have achieved a CCyR. However, 19 months after commencing treatment, he showed a significant increase in the BCR-ABL mRNA level (BCR-ABL:ABL ratio, 55\%) and was diagnosed with a cytogenetic relapse. Imatinib was discontinued, and he started therapy with nilotinib at a dose of 400 mg twice daily (Novartis US). However, 2 months later, progression to the blast phase (BP; hematologic and cytogenetic relapse) occurred, with detection of the kinase domain mutations M244V and $\mathrm{Y} 253 \mathrm{H}$ His peripheral blood WBC count increased to $69.45 \times 10^{9} / \mathrm{L}$ with $58 \%$ blasts, and bone marrow cytogenetics revealed $\mathrm{Ph}$-positive cells in all metaphases analyzed. The patient was then administered a course of daunorubicin (60 mg i.v. on days 1 to 3 , Pfizer US) and cytarabine (200 mg on days 1 to 7, Pharmacia Italy) together with imatinib 600 mg daily. However, the effects were unsatisfactory, and the percentage of blasts in the peripheral blood increased to $79 \%$. In November 2011, he commenced treatment with $140 \mathrm{mg}$ /day dasatinib (BristolMyers Squibb Company, US). After only 1 week of this treatment, his peripheral blood WBC count decreased to $1.14 \times 10^{9} / \mathrm{L}$, and a major Cytogenetic Response (MCyR) was achieved after 5 months of treatment $(\mathrm{BCR} / \mathrm{ABL}$ fusion gene detected in $1.3 \%$ of cells on FISH, and 500 white blood cells were obtained). He then underwent a halfmatched, related-donor, Stem Cell Transplantation (SCT) in May 2012;

*Corresponding author: Jia-Hua Ding, MD, PhD, Department of Hematology, Affiliated Zhongda Hospital Medical College of Southeast University, Ding Jia Qiao Number 76, Nanjing, 210009, China, Tel: 8625 83262460; Fax: 862583262461 E-mail: zll313628@sina.com

Received June 27, 2017; Accepted July 29, 2017; Published July 31, 2017

Citation: Yu Z, Ding J, Sun A, Ge Z, Chen B, et al. (2017) Second Generation Tyrosine Kinase Inhibitors Combined With Allogeneic Hematopoietic Stem Cell Transplantation Improve the Prognosis of Patients with Chronic Myelogenous Leukemia in Blast Crisis. J Stem Cell Res Ther 7: 393. doi: 10.4172/2157-7633.1000393

Copyright: (C) 2017 Yu Z, et al. This is an open-access article distributed unde the terms of the Creative Commons Attribution License, which permits unrestricted use, distribution, and reproduction in any medium, provided the original author and source are credited. 
Citation: Yu Z, Ding J, Sun A, Ge Z, Chen B, et al. (2017) Second Generation Tyrosine Kinase Inhibitors Combined With Allogeneic Hematopoietic Stem Cell Transplantation Improve the Prognosis of Patients with Chronic Myelogenous Leukemia in Blast Crisis. J Stem Cell Res Ther 7: 393. doi: 10.4172/2157-7633.1000393

Page 2 of 6

the $4.63 \times 10^{6} / \mathrm{kg} \mathrm{CD} 34+$ cells from his mother and transplanted, and the $\mathrm{BuCy}$ (the dose of busulfan $0.8 \mathrm{mg} / \mathrm{kg}$ was administered every $6 \mathrm{~h}$ for a total of 12 doses, Otsuka Pharmaceutical, Japan. Cyclophosphamide $60 \mathrm{mg} / \mathrm{kg} /$ day were administered intravenously for 2 days following the completion of the busulfan treatment, Shanghai Institute of Health Industry. simustine (MeCCNU, $250 \mathrm{mg} / \mathrm{m}^{2}$, a single dose oral. Swiss Pharmaceutical, Zhejiang). Conditioning regimen was used when cells were transplanted.

Patient 2: A 52-year-old man was diagnosed with CP CML in 2000. He was initially treated with hydroxyurea, and in April 2002, underwent HLA-identical, sibling, HSCT; the donor was his younger brother. However, he continued to have pancytopenia after the transplantation, and FISH revealed the BCR/ABL fusion gene in 5\% of cells. Chimerism analysis showed mixed chimerism. He underwent splenectomy [6], and 6 months later, his complete blood count gradually returned to normal. Moreover, the BCR/ABL fusion gene was now undetectable on FISH. Chromosome analysis of a bone marrow aspirate showed complete donor conversion. Thus, the patient achieved a CCyR. However, in June 2011, his WBC and platelet counts increased to $17.48 \times 10^{9} / \mathrm{L}$ and $1801 \times 10^{9} / \mathrm{L}$, respectively, with a bone marrow aspirate showing $11.6 \%$ myeloid blasts. He also had cytogenetic evidence of the t $(9 ; 22)$ $(\mathrm{q} 34 ; \mathrm{q} 11)$ translocation and progressed to the accelerated phase (AP). He was treated with interferon- $\alpha$ at a dose of 3,000,000 IU twice weekly, but without satisfactory effects. In July 2011, he received $600 \mathrm{mg} /$ day imatinib but progressed to $\mathrm{BC}$, and FISH showed that the BCR/ ABL fusion gene was present in $45.6 \%$ cells, while chimerism analysis showed that the percentage of donor genes was 19\%. In December 2011, treatment with nilotinib was commenced at a dose of $400 \mathrm{mg}$ twice daily, and an MCyR was achieved after only 3 months of treatment (BCR/ABL fusion gene detected in $5.3 \%$ cells on FISH). Fortunately, the patient had a second opportunity to undergo to a matched, unrelated-donor SCT in March 2012. the number of CD $34+$ cells $5.38 \times 10^{6} / \mathrm{kg}$ were transplanted, and the patient was discharged after hematopoietic recovery.

Patient 3: A 36-year-old man was diagnosed with CP CML in 2000. He was initially treated with interferon- $\alpha$ and imatinib for 2 years, and achieved a partial cytogenetic response (PCyR). However, in 2005, he progressed to the AP. Later that year, he underwent an 8-of-10 HLAmatched, unrelated-donor, HSCT. The $5.96 \times 10^{6} / \mathrm{kg} \mathrm{CD} 34^{+}$and 29.87 $\times 10^{9} / \mathrm{L}$ white blood cells were obtained and transplanted. Graft versus host disease (GVHD) prophylaxis consisting of cyclosporine and dexamethasone was administered. Nevertheless, he developed a grade 4 , acute GVHD of the skin, and finally recovered after treatment with cyclosporine, dexamethasone and immunoglobulin. He also developed chronic GVHD of the liver, kidney and eyes. In April 2012, his WBC and platelet counts increased to $19.14 \times 10^{9} / \mathrm{L}$ and $939 \times 10^{9} / \mathrm{L}$ respectively, with a bone marrow aspirate showing $21 \%$ myeloid blasts. FISH detected the BCR/ABL fusion gene in $78 \%$ cells. Chimerism analysis showed that the percentage of donor genes was $19 \%$. The patient progressed to the $\mathrm{BC}$, and was treated with $100 \mathrm{mg} /$ day dasatinib.

Patient 4: A 33-year-old woman diagnosed with CP CML in her 29th week of gestation in May 2011. A complete blood count showed the following: WBC count, $518.4 \times 10^{9} / \mathrm{L}$ with $5 \%$ blasts; hemoglobin, $6.0 \mathrm{~g} / \mathrm{dl}$; and platelets, $383 \times 10^{9} / \mathrm{L}$. Bone marrow aspirate examination revealed $1.5 \%$ myeloid blasts. FISH showed the BCR/ABL fusion gene in $88.6 \%$ of cells and a possible deletion of the ASS gene. The patient was initially treated with leukapheresis and hydroxyurea. One month later, her peripheral blood WBC count decreased to $1.14 \times 10^{9} / \mathrm{L}$, while her platelet count increased to $619 \times 10^{9} / \mathrm{L}$. At this stage, she opted for an induced abortion. Two months after the initial diagnosis, she underwent an allo-HSCT; the donor was her HLA-identical sister. the $4.97 \times 10^{6} / \mathrm{kg}$ CD34+ cells were obtained and transplanted, and the BuCy conditioning regimen was used when cells were transplanted (BuCy: the dose of busulfan $0.8 \mathrm{mg} / \mathrm{kg}$ was administered every $6 \mathrm{~h}$ for a total of 12 doses. Cyclophosphamide $60 \mathrm{mg} / \mathrm{kg} /$ day were administered intravenously for 2 days following the completion of the busulfan treatment, simustine MeCCNU, $250 \mathrm{mg} / \mathrm{m}^{2}$, a single dose oral). One month after the transplantation, CCyR was achieved; the BCR/ABL fusion gene was undetectable on FISH, and chimerism analysis showed complete donor conversion. However, in April 2012, she developed BC, and the bone marrow aspirate showed 30\% myeloid blasts. FISH detected the BCR/ ABL fusion gene in $80 \%$ cells, and chimerism analysis of the bone marrow showed 30\% donor genes. In May 2012, she began treatment with nilotinib at a dose of $200 \mathrm{mg}$ twice daily, which was subsequently reduced to $300 \mathrm{mg} /$ day due to intolerance. The characteristics of the patients are listed in Table 1.

\section{Donor hematopoietic stem cell mobilization and isolation}

Using G-CSF $(10 \mu \mathrm{g} / \mathrm{kg} \bullet \mathrm{d})$ the mobilization of peripheral blood stem cell donor. Monitoring of blood cell every day, in the fifth days on acquisition. Using COBE SpectraTM blood cell separator machine, Auto-PBSC collection procedures (software version 6.1) were apheresis. Femoral venous access catheter for blood collection, held until the end of the collection. Treatment volume was 2 times the total blood volume, flow rate of $35 \sim 50 \mathrm{ml} / \mathrm{min}$, with ACD-A solution as an anticoagulant, the blood flow velocity and the ratio of (10 to 12): 1 . Acquisition time of 200 to 300 minutes, collecting about $200 \mathrm{ml}$. Surgery for oral or intravenous calcium supplements to prevent hypocalcemia caused by anticoagulants. Flow chamber and sent for collection of blood cell morphology analysis room count, when gathering material mononuclear cells $(5.0 \sim 8.0) \times 10^{8} / \mathrm{kg}$, CD $34+$ cells $(2.0 \sim 4.0) \times 10^{6} / \mathrm{kg}$ the end of with the collection. The peripheral blood stem cells rapidly through the patient's jugular vein catheter quickly infusion to the patient, before reinfusion give $5 \%$ sodium bicarbonate $150 \mathrm{ml}$ and dexamethasone 2.5 $\mathrm{mg}$ intravenous drip.

\section{Donor hematopoietic stem cell transplantation}

Four patients diagnosed CML in blastic crisis were treated with allogeneic peripheral blood stem cell transplantation in this study. The busulfan and cyclophosphamide (BU-CY) conditioning regimen consisted of busulfan $(0.8 \mathrm{mg} / \mathrm{kg})$ administered every $6 \mathrm{~h}$ for 12 days and cyclophosphamide (CTX) $(60 \mathrm{mg} / \mathrm{kg})$ administered once a day for 2 days. Each donor received granulocyte colony stimulat- ing factor $(\mathrm{G}-\mathrm{CSF})(300 \mu \mathrm{g})$ twice a day for 5 days for mobilization. A median of $12 \mathrm{~L}$ (3 times the total blood volume) of donor blood was processed and collected. Each recipient received monocyte count (MNC) $8 \pm 2 \times$ $10^{8} / \mathrm{kg}$ and/or $5 \pm 1 \times 10^{6} / \mathrm{kg} \mathrm{CD} 34^{+}$cells of body weight. All patients and their donors gave written informed consent, and this study was approved by Institutional Review Board and the Ethics Committee at Southeast University.

\section{GVHD prophylaxis}

All patients received immunosuppressive therapy before allo-HSCT.

\begin{tabular}{|c|c|c|c|c|}
\hline Patients & Patient 1 & Patient 2 & Patient 3 & Patient 4 \\
\hline age & 28 & 52 & 36 & 33 \\
\hline BCR/ABL (Fish) before treatment & $55 \%$ & $45.6 \%$ & $78 \%$ & $80 \%$ \\
\hline BCR/ABL (Fish) after treatment & $1.3 \%$ & $5.3 \%$ & 0 & 0 \\
\hline BCR/ABL (PCR) before treatment & $42 \%$ & $32 \%$ & $35 \%$ & $47 \%$ \\
\hline BCR/ABL (PCR) after treatment & $0.53 \%$ & $1.2 \%$ & $0.01 \%$ & $0.01 \%$ \\
\hline
\end{tabular}

Table 1: Characteristics of patients, TKIs-II combined with allo-HSCT. 
Citation: Yu Z, Ding J, Sun A, Ge Z, Chen B, et al. (2017) Second Generation Tyrosine Kinase Inhibitors Combined With Allogeneic Hematopoietic Stem Cell Transplantation Improve the Prognosis of Patients with Chronic Myelogenous Leukemia in Blast Crisis. J Stem Cell Res Ther 7: 393. doi: $10.4172 / 2157-7633.1000393$

Page 3 of 6

Cyclosporin A (CsA) and methotrexate (MTX) were administered as a GVHD prophylaxis treatment. The regimen consisted of $3 \mathrm{mg} / \mathrm{kg}$ of intravenous CsA with a target blood level of $200-400 \mathrm{ng} / \mathrm{L}$ and a short course treatment of MTX (15 mg on day 1 and $10 \mathrm{mg}$ on days 3, 6, and 11 , respectively). If GVHD was successfully treated at that point, the dose was gradually reduced, and ended on the sixth month.

\section{Engraftment and chimerism analysis}

The neutrophil engraftment start point was defined as the first day of 3 consecutive days when the absolute neutrophil count (ANC) exceeded $0.5 \times 10^{9} / \mathrm{L}$. The platelet engraftment start point was defined as the first day of 3 consecutive days when the platelet count exceeded $20 \times 10^{9} / \mathrm{L}$ independently without a platelet transfusion. Donor cell engraftment was assessed in Peripheral Blood (PB) nucleated cells by quantitative Polymerase Chain Reaction (PCR) of variable numbers of tandem repeats, blood cell counts, Bone Marrow (BM) examination, cytogenetic and molecular analysis.

\section{Result}

One month after the transplantation, the patient 1 showed a Complete Molecular Response (CMR) and has remained stable for 22 months without dasatinib, BCR/ABL fusion gene detected in $0 / 500$ cells on FISH, RT-PCR was $0 \%$. Two months after this, the patient 2 achieved a CCyR, and the BCR/ABL fusion gene was undetectable on FISH after transplanation two months. Three months after the transplantation, he achieved a CMR and has remained progression free and BCR/ABL fusion gene negative for 23 months. The patient 3 was treated with dasatinib. After 1 month of treatment, his complete blood count returned to normal. After 2 months, he achieved an MCyR, and the BCR/ABL fusion gene was detected in only $4.0 \%$ of cells on FISH. After 3 months, he achieved a CCyR, and the BCR/ABL fusion gene was detectable $0 / 500$ on FISH, RT-PCR was $0.01 \%$. At this stage, chromosome analysis of the bone marrow showed complete donor conversion. The patient has been in a stable condition for 21 months, and the BCR/ABL fusion gene has remained undetectable on FISH. In May 2012, the patient 4 began treatment after 2 weeks, her peripheral blood cell counts had returned to normal. One month later, she achieved a CCyR, with $0 \%$ myeloid blasts in the bone marrow. The BCR/ABL fusion gene was detect abl 0/500 on FISH, RT-PCR was undetectable and chimerism analysis showed complete donor conversion. She has been stable and progression free achieved a durable 25 month follow-up.

\section{Discussion}

Presentation in $\mathrm{BC}$ is uncommon but carries a poor prognosis. $\mathrm{BC}$ is less responsive to conventional chemotherapy, and the postHSCT survival rate among patients with $\mathrm{BC}$ is low due to high relapse rates and treatment-related mortality. Data on transplantation during $\mathrm{BC}$ are discouraging. The European Group for Blood and Marrow Transplantation (EBMT) [7] analyzed 167 CML-BC patients who had undergone allo-HSCT between 1980 and 1990. They found that the 2-, 5-, 10- and 15-year Overall Survival (OS) rates were 21\%, 18\%, 16\% and $14 \%$, respectively [7].

Some studies have suggested that imatinib has no deleterious or beneficial effect on transplant outcomes [8]. Lee et al. analyzed $216 \mathrm{IM}^{+}$ and $216 \mathrm{IM}^{-}$allograft recipients with advanced-phase CML suggested that the use of imatinib before HSCT was not associated with treatmentrelated mortality, relapse, leukemia-free survival or overall survival [9].

Dasatinib is a multitargeted tyrosine kinase inhibitor of BCR$\mathrm{ABL}$ and SRC Family Kinases (SFKs). It effectively inhibits the active and inactive conformations of BCR-ABL and also inhibits the SFKs implicated in imatinib resistance. In vitro studies have shown that dasatinib has 325 -fold greater potency than imatinib in cells transduced with unmutated BCR-ABL and is active against all tested BCR-ABL mutations, except one (T315I), that confer imatinib resistance [10]. In a phase II clinical trial [3], dasatinib was administered to patients with imatinib-resistant and imatinib-intolerant CML-BC. The results of an 8-month follow-up showed that dasatinib induced major hematologic responses in 34\% Myeloid BP (MBP)-CML and 31\% lymphoid BP (LBP)-CML patients, MCyR in 31\% MBP-CML and 50\% LBP-CML patients and CCyR in 26\% MBP-CML and 43\% LBP-CML patients. The myeloid BC (MBC)-CML and Lymphoid BC (LBC)-CML patients who achieved major hematologic responses had not experienced disease progression at the 8-month follow-up [3]. These outcomes were similar to those of another international, phase II clinical trial [11], in which dasatinib was administered orally (70 mg twice daily) to $10^{9} \mathrm{MBP}-\mathrm{CML}$ and 48 LBP-CML patients. After a minimum follow-up of 12 months, major hematologic responses were induced in 34\% MBP-CML and 35\% LBP-CML patients. MCyR was attained in 33\% MBP-CML and 52\% LBP-CML patients, while CCyR was attained in 26\% MBP-CML and $46 \%$ LBP-CML patients. The median progression-free survival was 6.7 months in MBP-CML patients and 3.0 months in LBP-CML patients. The median OS was 11.8 months in MBP-CML patients and 5.3 months in LBP-CML patients [11].

TKIs-II can produce a rapid and significant effect in CML BC. CML patients undergoing allo-HSCT may receive a TKI-II (dasatinib or nilotinib) after failure of imatinib treatment. Jabbour $\mathrm{E}$ analyzed the outcomes of 12 patients, most of whom had advanced CML (AP, 6; BP, 5) and who had received dasatinib $(n=2)$, nilotinib $(n=7)$ or both $(n=3)$ before undergoing HSCT. Of the 12 patients, nine achieved a molecular response, including four complete and five major responses. After a median follow-up of 10 months, seven patients were alive and showed a molecular response, while five patients had died, including four who died of disease progression [5]. The authors concluded that previous treatment with a TKI-II did not increase transplant-related toxicity. A high rate of molecular responses was achieved after HSCT, despite the fact that all patients underwent transplantation while they were in an imatinib-refractory, advanced disease stage [5]. TKIs-II have the potential advantage of rescuing patients after failure of imatinib therapy, and allowing these patients to undergo transplantation with a smaller disease bulk, which should improve disease control after HSCT [5]. Breccia et al. administered pre-SCT dasatinib treatment to two CML-BC patients (treatment duration, 8 and 6 months), who achieved a CCyR and a CHR [12]. Similarly, Gotoh et al. [13]. reported the case of a patient with imatinib- and nilotinib-resistant, Ph-positive CML-BC. Before dasatinib therapy, this patient was found to have a F359V BCRABL mutation. He was treated with dasatinib before allo-HSCT, and eventually achieved molecular remission [13].

The GVL effect is believed to destroy leukemia cells through an antitumor T-cell and natural killer (NK)-cell immune response. A mechanism has been proposed to account for antitumor T-cell and NKcell dysfunction: secretion of suppressive factors such as transforming growth factor $\beta 1[14,15]$ and interleukin $10[16]$, in the leukemia microenvironment. Thus, for a lower leukemia burden, the GVL effect will be greater due to the less stringent downregulation of the antitumor T-cell immune response mediated by the decreased secretion of suppressive factors. Some reports have suggested that high-dose chemotherapy induced severe cytoreduction, allowing the development of a more potent GVL effect $[17,18]$. A recent study has indicated that the achievement of a MCyR before allo-HSCT may improve the 
Citation: Yu Z, Ding J, Sun A, Ge Z, Chen B, et al. (2017) Second Generation Tyrosine Kinase Inhibitors Combined With Allogeneic Hematopoietic Stem Cell Transplantation Improve the Prognosis of Patients with Chronic Myelogenous Leukemia in Blast Crisis. J Stem Cell Res Ther 7: 393. doi: 10.4172/2157-7633.1000393

Page 4 of 6

prognosis of CML-BC patients [19]. Another report has indicated that dasatinib might enhance the GVL effect [20].

In our study, patients 1 and 2 received imatinib during $\mathrm{BC}$ or $\mathrm{AP}$, but did not achieve remission or became worse. However, both patients achieved MCyR soon after their treatment regimen was changed to TKIs-II. With a smaller disease bulk, the subsequent allo-HSCT induced CMR in both patients (after 1 and 3 months). Both patients are alive and disease free survival at 22 and 23 months after allo-HSCT. It has become clear that high-dose chemotherapy does not eradicate malignancy in many CML patients and that the therapeutic benefit of allo-HSCT is largely related to an associated immune-mediated, GRaft-VersusLeukemia (GVL) effect. Compared with imatinib, TKIs-II may induce cytogenetic or molecular responses more quickly and more effectively in CML patients $[21,22]$, reducing the pretransplant tumor burden to a greater degree [23]. Therefore, we speculate that TKIs-II can reduce the pretrans- plant tumor burden to a greater degree and enhance the effect of GVL (Figure 1), GVL and tumor burden are negatively correlated. Consequently, the success rate of allo-HSCT may be increased, and the relapse rate may be reduced.

The prognosis of patients who relapse into BC after allo-HSCT is very poor. Donor lymphocyte infusion (DLI) is considered the standard management for CML relapse after HSCT, but this treatment has very limited success when administered to CML patients who relapse with BC $[24,25]$. Imatinib is now an alternative to DLI in the management of patients relapsing after transplantation, but this treatment also has a poor outcome in patients who relapse with BC. Recently, the Chronic
Leukemia Working Party of the EBMT reported a retrospective analysis of 128 patients treated with imatinib for relapse after allogeneic transplantation. The CCyR was $22 \%$ for the patients in $\mathrm{BC}$, at a median follow-up of 9 months; the estimated 2-year survival for BC patients was only $12 \%$ [26]. DeAngelo et al. administered imatinib to $15 \mathrm{CML}$ patients who relapsed after transplantation, including four patients in the AP and one in the BP. Only one of these patients achieved CMR (Figure 2) [27].

In the current study, both patients 3 and 4 underwent allo-HSCT and received TKIs-II after they relapsed with BC. CHR was achieved in several weeks, and CCyR was achieved after 3 months and 1 month in patients 3 and 4, respectively. In both patients, chromosome analysis of the bone marrow showed complete donor conversion. In patient 3 , we observed that the chronic GVHD was alleviated during the recurrence of CML. As the CML was treated, the cGVHD re-appeared. Both patients are alive and remain progression free after 21 and 22 months of follow-up.

Several factors might have contributed to our superior TKIs-II combined with transplantation outcomes. First, the initial reduction of the leukemia load via short-term treatment with TKIs-II as a bridging therapy seemed to result in lower transplantation-related mortality and better chances of long-term survival. Secondly, BC patients achieved a second CP with short-term TKIs-II therapy before transplantation with a speed reduced disease burden. Thirdly, TKIs-II combined with HSCT by minimal residual disease levels might have contributed to decrease relapse and improve survival. In addition, TKIs-II combined with allo-

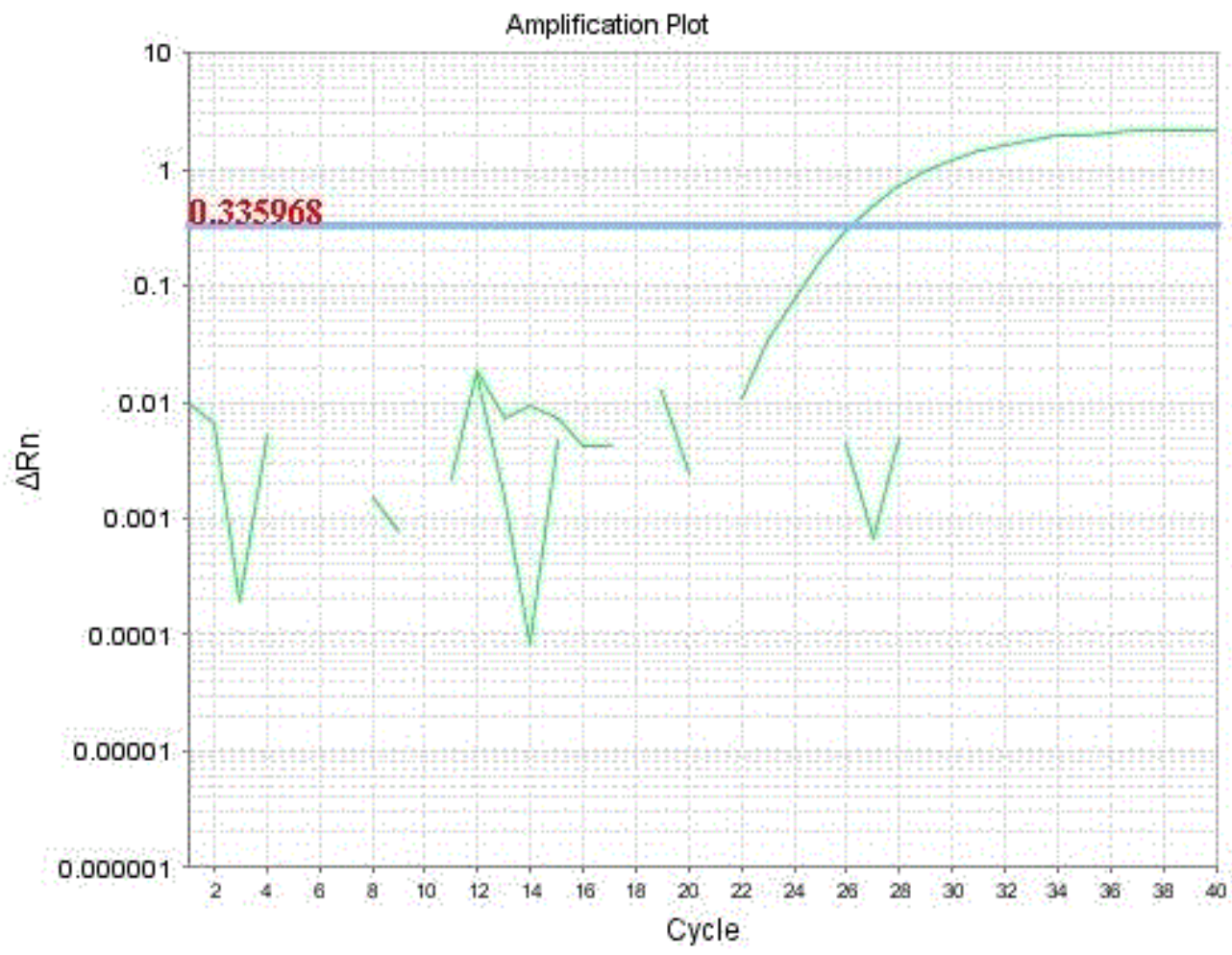

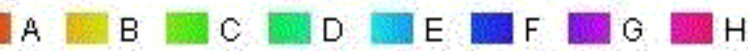

Figure 1: The BCR-ABL:ABL ratio detected by reverse transplantation polymerase chain reaction (RT-PCR after transplantation). 
Citation: Yu Z, Ding J, Sun A, Ge Z, Chen B, et al. (2017) Second Generation Tyrosine Kinase Inhibitors Combined With Allogeneic Hematopoietic Stem Cell Transplantation Improve the Prognosis of Patients with Chronic Myelogenous Leukemia in Blast Crisis. J Stem Cell Res Ther 7: 393. doi: $10.4172 / 2157-7633.1000393$

Page 5 of 6

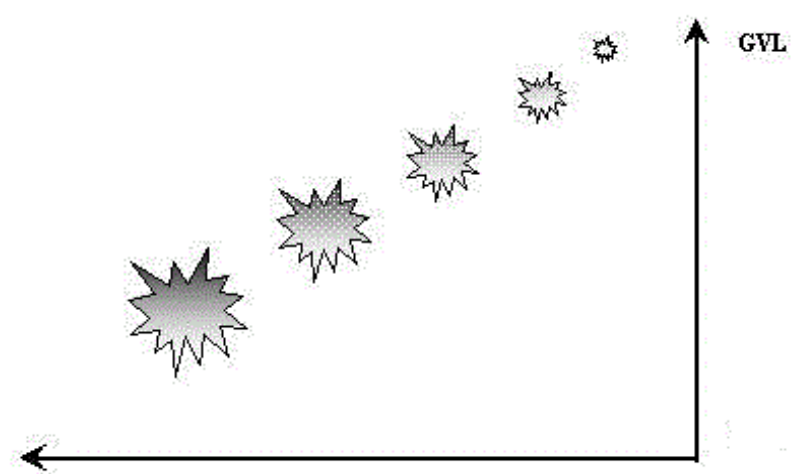

Tumor burden

Figure 2: The relationship between GVL and Tumor burden.

HSCT also delivered a higher quality of molecular response than TKIsII alone.

It has been suggested that the GVL effect is more likely to occur after the achievement of complete donor chimerism [28]. Compared with imatinib, TKIs-II can eliminate leukemic clones resistant to the GVL effect in a shorter time, reducing disease burden to levels below cytogenetic or molecular detection more quickly. The GVL effect mediated by antitumor T-cell and NK-cell immune responses may be re-induced because of the decreased secretion of suppressive factors. An efficient GVL effect may durably suppress residual CML progenitors and reduce recurrence rates. Thus, we suggest that compared with imatinib therapy, early use of TKIs-II may quickly and effectively induce a more long-lasting remission in patients who have relapsed with BC after HSCT. We speculate that the GVL effect may be reinduced because of the decreased disease bulk.

\section{Conclusion}

Compared with imatinib, TKIs-II may reduce tumor burden more quickly and to a greater extent (a cytogenetic or molecular remission) and enhance the GVL effect. This enhanced effect may in turn increase the success rate of allo-HSCT and reduce recurrence. DLI has a very limited success in the treatment of CML patients who relapse with BC after allo-HSCT. In such patients, the early use of TKIsII can eliminate the residual leukemic clones, and reduce the disease burden to levels below cytogenetic or molecular detection. The GVL effect mediated by antitumor T-cell and NK-cell immune responses may be re-induced. This enhanced GVL effect may durably suppress residual CML progenitors after HSCT and reduce recurrence rates. Therefore, we suggest that the reduction of tumor burden using secondgeneration tyrosine kinase inhibitors, in combination with stem cell transplantation, favorably alters the prognosis of CML in BC. Further well-designed, randomized studies involving a large number of patients are required to evaluate this strategy.

\section{Author Contribution}

Jiahua Ding designed and performed the research. Analyzed the data and wrote the paper with Zhengping Yu.

\section{Conflict of Interest}

The authors declare no competing financial interests.

\section{References}

1. Sawyers CL, Hochhaus A, Feldman E, Goldman JM, Miller CB, et al. (2002)
Imatinib induces hematologic and cytogenetic responses in patients with chronic myelogenous leukemia in myeloid blast crisis: results of a phase II study. Blood 99: 3530-3539. [PubMed]

2. Khoury HJ, Kukreja M, Goldman JM, Wang T, Halter J, et al. (2012) Prognostic factors for outcomes in allogeneic transplantation for CML in the imatinibera: a CIBMTR analysis. Bone Marrow Transplantation 47: 810-816. [PubMed]

3. Cortes J, Rousselot P, Kim DW, Ritchie E, Hamerschlak N, et al. (2007) Dasatinib induces complete hematologic and cytogenetic responses in patients with imatinib-resistant or intolerant chronic myeloid leukemia in blast crisis. Blood 109: 3207-3213. [PubMed]

4. Saglio G, Hochhaus A, Goh YT, Masszi T, Pasquini R, et al. (2010) Dasatinib in imatinib-resistant or imatinib-intolerant chronic myeloid leukemia in blast phase after 2 years of follow-up in a phase 3 study: efficacy and tolerability of 140 milligrams once daily and 70 milligrams twice daily. Cancer 116: 3852-3861. [PubMed]

5. Jabbour E, Cortes J, Kantarjian H, Giralt S, Andersson BS, et al. (2007)Nove tyrosine kinase inhibitor therapy before allogeneic stem cell transplantation in patients with chronic myeloid leukemia: no evidence for increased transplantrelated toxicity. Cancer 110: 340-344. [PubMed]

6. Ding J, Bao W, Wang J, Zhao G, Chen J, et al. (2011) Effect of Splenectomy on Pancytopenia After a Peripheral Blood Stem Cell Transplantation. Cell Transplant 20: 1109-1115. [PubMed]

7. Gratwohl A, Brand R, Apperley J, Crawley C, Ruutu T, et al. (2006) Allogeneic hematopoietic stem cell transplantation for chronic myeloid leukemia in Europe 2006: transplant activity, long-term data and current results. An analysis by the Chronic Leukemia Working Party of the European Group for Blood and Marrow Transplantation (EBMT). Haematologica 91: 513-521. [PubMed]

8. Saussele S, Lauseker M, Gratwohl A, Beelen DW, Bunjes D, et al. (2010) Allogeneic hematopoietic stem cell transplantation (allo SCT) for chronic myeloidleukemia in the imatinib era: evaluation of its impact within a subgroup of the randomized German CML Study IV. Blood 2010; 115: 1880-1885. [PubMed]

9. Lee SJ, Kukreja M, Wang T, Giralt SA, Szer J, et al. (2008) Impact of prio imatinib mesylate on the outcome of hematopoietic cell transplantation for chronic myeloid leukemia. Blood 112: 3500 -3507. [PubMed]

10. Müller MC, Cortes JE, Kim DW, Druker BJ, Erben P, Pasquini R, et al. (2009) Dasatinib treatment of chronic-phase chronic myeloid: analysis of responses according to preexisting BCR-ABL mutations. Blood 114: 4944-4953. [PubMed]

11. Cortes J, Kim DW, Raffoux E, Martinelli G, Ritchie E, et al. (2008) Efficacy and safety of dasatinib in imatinib-resistant or -intolerant patients with chronic myeloid leukemia in blast phase. Leukemia 22: 2176-2183.

12. Breccia M, Palandri F, lori AP, Colaci E, Latagliata R, et al. (2010) Secondgeneration tyrosine kinase inhibitors before allogeneic stem cell transplantation in patients with chronic myeloid leukemia resistant to imatinib. Leuk Res 34: 143-147. [PubMed]

13. Gotoh M, Tauchi T, Yoshizawa S, Kitahara T, Kiguchi T, et al. (2010) Successful prior treatment with dasatinib followed by stem cell transplantation in a patien with CML in blastic crisis with a BCR-ABL mutation. Int J Hematol 91: 128-131. [PubMed]

14. Inge TH, Hoover SK, Susskind BM, Barrett SK, Bear HD (1992) Inhibition of tumorspecific cytotoxic T-lymphocyte responses by transforming growth factor beta 1. Cancer Res 52: 1386-1392.

15. Ruffini PA, Rivoltini L, Silvani A, Boiardi A, Parmiani G (1993) Factors including transforming growth factor $\beta$, released in the glioblastoma residual cavity, impair activity of adherent lymphokine-activated killer cells. Cancer Immunol Immunother 36: 409-416.

16. Matsuda M, Salazar F, Petersson M, Masucci G, Hansson J, et al. (1994) Interleukin 10 pretreatment protects target cells from tumor and allo-specific cytotoxic T cells and downregulates HLA class I expression. J Exp Med 180: 2371-2376. [PubMed]

17. Giralt S, Thall PF, Khouri I, Wang X, Braunschweig I, et al. (2001) Melphalan and purine analog-containing preparative regimens:reduced-intensity conditioning for patients with hematologic malignancies undergoing allogeneic progenitor cell transplantation. Blood 97: 631-637. [PubMed]

18. Khouri IF, Saliba RM, Giralt S (2001) Long-term remission and low mortality achieved with cisplatin,fludarabine,cytarabine nonablative preparative regmen 
Citation: Yu Z, Ding J, Sun A, Ge Z, Chen B, et al. (2017) Second Generation Tyrosine Kinase Inhibitors Combined With Allogeneic Hematopoietic Stem Cell Transplantation Improve the Prognosis of Patients with Chronic Myelogenous Leukemia in Blast Crisis. J Stem Cell Res Ther 7: 393. doi: $10.4172 / 2157-7633.1000393$

Page 6 of 6

and allogeneic stem cell transplantation for histologically aggressive nonHodgkin's lymphoma. Blood.

19. Weisser M, Schleuning M, Haferlach C, Schwerdtfeger R, Kolb HJ (2007) Allogenetic stem-cell transplantation provides excellent results in advanced stage chronic myeloidleukemia with major cytogenetic response to pretransplant imatinib therapy. Leuk Lymphoma 48: 259-301. [PubMed]

20. Garland P, Dazzi F, Marin D (2010) Dasatinib may not suppress the GVL effect of donor lymphocyte infusions for CML. Bone Marrow Transplant 45: 395-396.

21. Kantarjian H, Shah NP, Hochhaus A, Cortes J, Shah S, et al. (2010) Dasatinib versus Imatinib in Newly Diagnosed Chronic-Phase Chronic Myeloid Leukemia. N Engl J Med 362: 2260-2270. [PubMed]

22. Saglio G, Kim DW, Issaragrisil S, le Coutre P, Etienne G, et al. (2010) ENESTnd Investigators. Nilotinib versus Imatinib for Newly Diagnosed Chronic Myeloid Leukemia. N Engl J Med 362: 2251-2259. [PubMed]

23. Baccarani M, Cortes J, Pane F, Niederwieser D, Saglio G, et al. (2009) Chronic myeloid leukemia: an update of concepts and management recommendations of European Leukemia Net. J Clin Oncol 27: 6041-6051.

24. Dazzi F, Szydlo RM, Craddock C, Cross NC, Kaeda J, et al. Comparison of single-dose and escalating-dose regimens of donor lymphocyte infusion for relapse after allografting for chronic myeloid leukemia. Blood 95: 67-71. [PubMed]

25. Shimoni A, Kröger N, Zander AR, Rowe JM, Hardan I, et al. (2003) Imatinib mesylate (STI571) in preparation for allogeneic hematopoietic stem cell transplantation and donor lymphocyte infusions in patients with Philadelphiapositive acute leukemias. Leukemia 17: 290-297. [PubMed]

26. Olavarria E, Ottmann OG, Deininger M, Clark RE, Bandini G, et al. (2003) Chronic Leukaemia Working Party of the European Group of Bone and Marrow Transplantation (EBMT). Response to imatinib in patients who relapse after allogeneic stem cell transplantation for chronic myeloid leukemia. Leukemia 17: $1707-1712$.

27. DeAngelo DJ, Hochberg EP, Alyea EP, Longtine J, Lee S, et al. (2004) Extended follow-up of patients treated with imatinib mesylate (gleevec) for chronic myelogenous leukemia relapse after allogeneic transplantation: durable cytogenetic remission and conversion to complete donor chimerism without graft-versus-host disease. Clin Cancer Res 10: 5065-5071. [PubMed]

28. Ueno NT, Cheng YC, Rondón G, Tannir NM, Gajewski JL, et al. (2003) Rapid induction of complete donor chimerism by the use of a reducedintensity conditioning regimen composed of fludarabine and reduced-intensity conditioning regimen composed of fludarabine and tumors. Blood 102: 38293836. [PubMed] 